\title{
Long-Term Cognitive Impairment after Hospitalization for Community-Acquired Pneumonia: a Prospective Cohort Study
}

\author{
Timothy D. Girard, MD, MSCl', Wesley H. Self, MD, MPH², Kathryn M. Edwards, $\mathrm{MD}^{3,4}$, \\ Carlos G. Grijalva, MD, MPH ${ }^{5,6}$, Yuwei Zhu, MD, $M S^{7}$, Derek J. Williams, MD, MPH ${ }^{3,8}$, Seema Jain, $M D^{9}$, \\ and James C. Jackson, Psy $D^{10,11,12,13}$
}

\begin{abstract}
${ }^{1}$ Clinical Research, Investigation, and Systems Modeling of Acute illness (CRISMA) Center in the Department of Critical Care Medicine, University of Pittsburgh School of Medicine, Pittsburgh, PA, USA; '2Department of Emergency Medicine, Vanderbilt University School of Medicine, Nashville, TN, USA; ${ }^{3}$ Vanderbilt Vaccine Research Program, Vanderbilt University School of Medicine, Nashville, TN, USA; ${ }^{4}$ Division of Pediatric Infectious Diseases, Vanderbilt University School of Medicine, Nashville, TN, USA; ${ }^{5}$ Department of Health Policy, Vanderbilt University School of Medicine, Nashville, TN, USA; ${ }^{6}$ Geriatric Research, Education and Clinical Center Service, Tennessee Valley Healthcare System, Nashville, TN, USA; ${ }^{7}$ Department of Biostatistics, Vanderbilt University School of Medicine, Nashville, TN, USA; ${ }^{8}$ Division of Hospital Medicine in the Department of Pediatrics, Vanderbilt University School of Medicine, Nashville, TN, USA; ${ }^{9}$ Influenza Division, National Center for Immunization and Respiratory Diseases, Centers for Disease Control and Prevention, Atlanta, GA, USA; ${ }^{10}$ Division of Allergy, Pulmonary, and Critical Care Medicine, Vanderbilt University School of Medicine, Nashville, TN, USA; ${ }^{11}$ Center for Health Services Research in the Department of Medicine, Vanderbilt University School of Medicine, Nashville, TN, USA; ${ }^{2}$ Department of Psychiatry, Vanderbilt University School of Medicine, Nashville, TN, USA; ${ }^{13}$ Research Service at the Department of Veterans Affairs Medical Center, Tennessee Valley Healthcare System, Nashville, TN, USA.
\end{abstract}

BACKGROUND: Recent studies suggest older patients hospitalized for community-acquired pneumonia are at risk for new-onset cognitive impairment. The characteristics of long-term cognitive impairment after pneumonia, however, have not been elucidated.

OBJECTIVE: To characterize long-term cognitive impairment among adults of all ages hospitalized for community-acquired pneumonia.

DESIGN: Prospective cohort study.

PARTICIPANTS: Adults without severe preexisting cognitive impairment who were hospitalized with communityacquired pneumonia.

MAIN MEASURES: At enrollment, we estimated baseline cognitive function with the Short Informant Questionnaire on Cognitive Decline in the Elderly (IQCODE). At 2- and 12month follow-up, we assessed cognition using the Repeatable Battery for the Assessment of Neuropsychological Status (RBANS) and tests of executive function, diagnosing cognitive impairment when results were $\geq 1.5$ standard deviations below published age-adjusted means for the general population. We also identified subtypes of mild cognitive impairment using standard definitions.

KEY RESULTS: We assessed 58 (73\%) of 80 patients who survived to 2-month follow-up and 57 (77\%) of 74 who survived to 12-month follow-up. The median [range] age of survivors tested was 57 [19-97] years. Only 8 (12\%) had evidence of mild cognitive impairment at baseline according to the Short IQCODE, but 21 (38\%) at 2 months and 17 (30\%) at 12 months had mild cognitive impairment per the RBANS. Moderate-to-severe cognitive impairment was common among adults $\geq 65$ years [4/13 (31\%) and

Electronic supplementary material The online version of this article (https://doi.org/10.1007/s11606-017-4301-x) contains supplementary material, which is available to authorized users.

Received October 26, 2017

Revised December 28, 2017

Accepted December 28, 2017

Published online January 26, 2018
$5 / 13(38 \%)$ at 2 and 12 months, respectivelyl but also affected many of those < 65 years [10/43 (23\%) and $8 / 43$ (19\%) at 2 and 12 months, respectively]. Deficits were most often noted in visuospatial function, attention, and memory.

CONCLUSIONS: A year after hospitalization for community-acquired pneumonia, moderate-to-severe impairment in multiple cognitive domains affected onethird of patients $\geq 65$ years old and $20 \%$ of younger patients, and another third of survivors had mild cognitive impairment.

KEY WORDS: pneumonia; cognition disorders; mild cognitive impairment; dementia; hospitalization.

J Gen Intern Med 33(6):929-35

DOI: $10.1007 / \mathrm{s} 11606-017-4301-\mathrm{x}$

(c) Society of General Internal Medicine 2018

$\mathrm{C}$ ommunity-acquired pneumonia (CAP) is a major source of morbidity and mortality. Each year, over a million hospitalizations in the US alone are attributable to this common infectious disease. ${ }^{1-3}$ Most studies of CAP outcomes have limited follow-up to in-hospital or 30-day outcomes, ${ }^{4}$ and the body of literature that has examined long-term outcomes after CAP has focused primarily on mortality. ${ }^{5-7}$ Yet, many patients who survive hospitalization for CAP struggle with diminished cognitive and functional abilities for prolonged periods after their acute illness. ${ }^{8-10}$

Numerous reports have shown that survivors of acute respiratory failure and sepsis, which are often caused by pneumonia, are at high risk for cognitive impairment that lasts years after discharge from the ICU and hospital. ${ }^{11-16}$ In addition, several recent studies found that older adults hospitalized for CAP, many of whom were admitted to general medical floors 
without overt organ failure, also experienced persistent declines in cognition. $^{8-10}$ These reports, however, were limited by their use of brief screening tests of cognition, which (unlike comprehensive cognitive batteries) are unable to identify the specific domains of cognition that are impaired in survivors of CAP. Additionally, these earlier studies focused specifically on older patients. Thus, it remains unknown whether younger adults who are hospitalized with CAP are also at risk for subsequent cognitive impairment.

We conducted the Cognitive Outcomes after CommunityAcquired Pneumonia (CogCAP) Study to determine the prevalence and severity of long-term cognitive impairment in adults up to 1 year after hospitalization for CAP. We hypothesized that long-term cognitive impairment in this population - the majority of which does not require intensive care - is common and affects multiple cognitive domains.

\section{METHODS}

\section{Study Design and Population}

We nested this single-center study within a larger prospective cohort study, the Centers for Disease Control and Prevention (CDC) Etiology of Pneumonia in the Community (EPIC) Study. ${ }^{17}$ The EPIC study was a multicenter investigation designed to determine the incidence and etiologies of CAP resulting in hospitalization in the US. From January 2010 to June 2012, patients admitted to the Vanderbilt University Medical Center were enrolled in the EPIC Study if they lived in the surrounding nine-county catchment area and had community-acquired pneumonia, defined as evidence of acute infection with signs/symptoms of acute respiratory illness and radiologic evidence of pneumonia, confirmed by a boardcertified chest radiologist who was unaware of the clinical data. Exclusion criteria included recent hospitalization; immunosuppression due to malignancy, recent organ or stem cell transplant, or human immunodeficiency virus infection with a CD4 cell count $<200 / \mathrm{mm}^{3}$; nursing home residence with functional dependence; or lack of informed consent, as previously described. ${ }^{17}$

Beginning in March 2011, adults enrolled in EPIC at Vanderbilt University were eligible for inclusion in the CogCAP Study if they survived to hospital discharge and had none of the following exclusion criteria: severe cognitive or neurodegenerative disease that prevented independent living prior to CAP (according to medical records and/ or an authorized representative); active substance abuse, psychotic disorder, homelessness, or plans to move out of the catchment area within 12 months of enrollment, any of which could prevent long-term follow-up; blindness or deafness, which would prevent outcomes assessment; lack of commitment to aggressive treatment (e.g., discharge to hospice); or lack of informed consent.

Patients were enrolled in the EPIC study within $24 \mathrm{~h}$ of hospital admission, but we did not enroll patients in CogCAP until 1 to 2 days prior to discharge, at which time we obtained separate written informed consent for CogCAP participation. The Vanderbilt University and CDC Institutional Review Boards approved the study protocol.

\section{Baseline and In-Hospital Assessments}

At enrollment, a trained psychology professional evaluated participants' baseline (i.e., pre-CAP hospitalization) cognitive and functional status using the following questionnaires, validated to assess pre-illness status. We assessed baseline global cognition with the Short Form of the Informant Questionnaire on Cognitive Decline in the Elderly (Short IQCODE), ${ }^{18}$ baseline activities of daily living (ADLs) with the Katz ADL questionnaire, ${ }^{19}$ and baseline instrumental ADLs with the Functional Activities Questionnaire (FAQ). ${ }^{20}$ Both participants (when able) and surrogates (e.g., a family member or close friend) completed questionnaires; if a questionnaire's score differed between the participant and surrogate, we used the worst score to maximize sensitivity.

We obtained demographic and clinical data from the medical record. These included data required to calculate the Charlson Comorbidity Index, ${ }^{21}$ which summarizes the total burden of comorbid illness prior to hospitalization by assigning points to specific chronic conditions (e.g., 1 point for congestive heart failure, 3 points for moderate-to-severe liver disease, and 6 points for metastatic solid malignancy), and the Pneumonia Severity Index, ${ }^{4}$ a severity of illness measure that has been validated in several community-acquired pneumonia cohorts.

\section{Outcomes}

Two and 12 months after hospital discharge, psychology professionals assessed participants' cognition using the Repeatable Battery for the Assessment of Neuropsychological Status (RBANS), ${ }^{22}$ a comprehensive, validated battery that evaluates five domains of cognitive function: immediate memory, delayed memory, attention, visuospatial construction, and language (described in detail in eTable 1). In addition, we also used the Mini-Mental State Examination (MMSE) to assess general cognition, the Trail Making Test $\mathrm{A}^{23}$ to assess attention, the Trail Making Test $\mathrm{B}^{23}$ and the Tower Test (a subtest of the Delis-Kaplan Executive Function System ${ }^{24}$ ) to assess executive function, the Katz Activities of Daily Living ${ }^{19}$ and the Functional Activities Questionnaire ${ }^{20}$ to assess activities of daily living and instrumental activities of daily living, and the Short $\mathrm{IQCODE}^{18}$ to assess self-reported changes in cognition.

\section{Statistical Analysis}

To determine whether patients who were tested during followup were different from those who were not, we used Fisher's exact tests to compare categorical variables and Wilcoxon rank-sum tests to compare continuous variables. 
We categorized patients as having mild cognitive impairment at baseline if either their patient-based or surrogate-based Short IQCODE was > 3.6; overall scores were obtained by averaging the scores of individual items. Following conventional neuropsychologic research methods, we compared participants' results for each test to published age-adjusted norms for the general population and considered results $\geq 1.5$ standard deviations below age-adjusted population means to indicate impairment. Per published norms, 6.7\% of the general population is expected to score below this cutoff. We identified mild cognitive impairment (which we subdivided into four mutually exclusive subtypes) using the standard definitions described in eTable $2,{ }^{25}$ and we defined "moderate-tosevere" cognitive impairment as that resulting in RBANS global index scores $<78$.

We used Stata Statistical Software (Release 10.1; StataCorp LP, College Station, TX) for all statistical analyses.

\section{RESULTS}

Between March 2011 and June 2012, 265 adults admitted to Vanderbilt University Medical Center with CAP were enrolled in the EPIC study. Of these, we enrolled 86 (32\%) in the CogCAP Study; 72 were not approached because of lack of staffing (e.g., they were discharged when CogCAP study personnel were not available), 63 declined to participate in the longterm study, and 44 were excluded for reasons shown in Fig. 1. Six $(7 \%)$ of the 86 patients enrolled in CogCAP died within 2 months of hospital discharge, and $7(8 \%)$ others died

265 patients enrolled in the EPIC Study
at Vanderbilt University from
March 2011 to June 2012

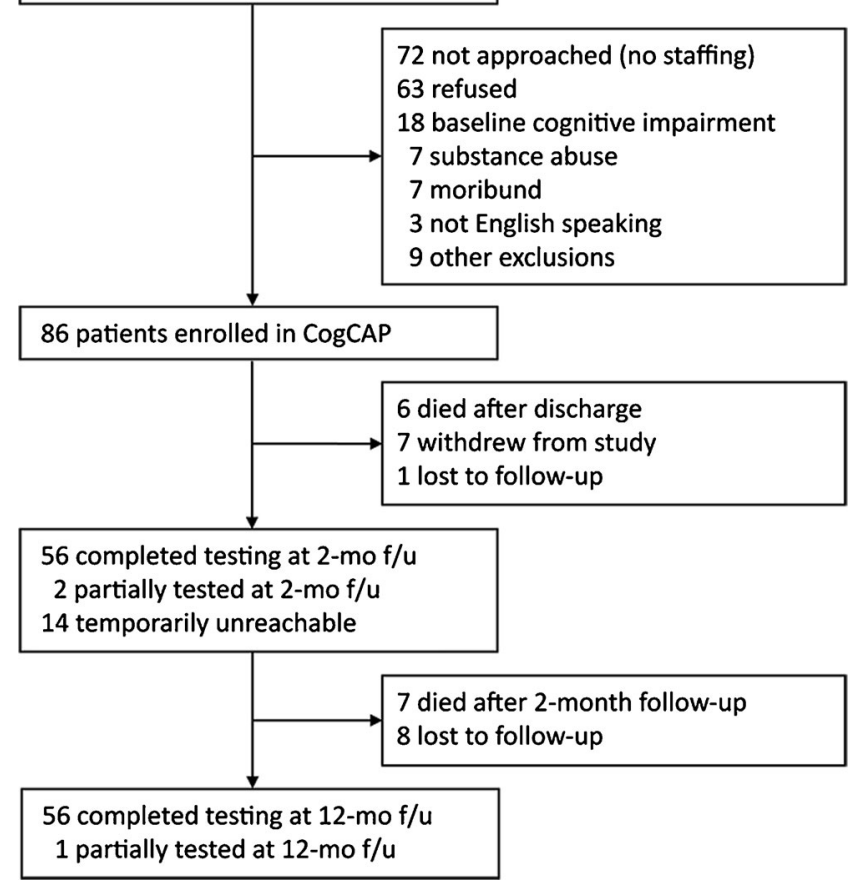

Figure 1 Enrollment and follow-up between 2 and 12 months, such that 80 subjects were eligible for follow-up at 2 months and 73 were eligible at 12 months. Of these, we assessed 58 (73\%) 2-month survivors and 57 (78\%) 12-month survivors. Participants who were alive but not assessed at 2 and/or 12 months were unable or unwilling to participate in the follow-up visit or were no longer reachable by study personnel.

The follow-up cohort included 67 subjects tested at 2 months and/or 12 months (Table 1). Patients with a broad range of ages were included, the median [interquartile] age being 57 [48-64] years and the range spanning from 19 to 97 years of age. Eighteen (27\%) subjects had no comorbid illness at baseline (Charlson Comorbidity Index $=0$ ), another $27 \%$ had only one comorbidity (Charlson Index $=1$ ), and $46 \%$ had multiple comorbidities (Charlson Index $\geq 2$ ). Though patients with moderate/severe preexisting cognitive impairment (e.g., neurodegenerative disease that prevented independent living) were excluded, those with mild cognitive impairment at time of CAP hospitalization were eligible, with eight (12\%) patients having a Short IQCODE > 3.6 suggesting some cognitive impairment at baseline.

Compared with those assessed at follow-up, patients who were never tested because of post-discharge death were more severely ill at admission per their Pneumonia Severity Index, ICU admission status, and need for invasive mechanical ventilation (eTable 3). Alternatively, patients who survived but were never tested (i.e., lost to follow-up) were not significantly different from those tested (eTable 4).

At 2-month follow-up, 14 (24\%) of 56 patients assessed had RBANS global cognition scores indicating moderate-tosevere cognitive impairment (Tables 2 and 3). In contrast, published norms indicate that $<7 \%$ of the general population has RBANS global cognition scores in this range. At 12 months, $13(23 \%)$ of 56 had scores consistent with moderate-to-severe cognitive impairment. At 2-month

Table 1 Characteristics of Patients Assessed 2 to 12 Months after Hospitalization with Community-Acquired Pneumonia

\begin{tabular}{|c|c|}
\hline Characteristic & No. $=67$ \\
\hline Age, median [IQR], years & $57[48-64]$ \\
\hline Female, no. (\%) & $40(60)$ \\
\hline \multicolumn{2}{|l|}{ Race, no. (\%) } \\
\hline White & $41(61)$ \\
\hline Black & $24(36)$ \\
\hline Other & $2(3)$ \\
\hline Charlson Comorbidity Index, median [IQR] & $1[0-3]$ \\
\hline Baseline cognitive impairment, no. (\%) & $8(12)$ \\
\hline \multicolumn{2}{|l|}{ Pneumonia Severity Index, no. $(\%)$} \\
\hline Low risk $(1-2)$ & $31(46)$ \\
\hline Moderate risk (3) & $19(28)$ \\
\hline High risk $(4-5)$ & $17(25)$ \\
\hline Presenting systolic blood pressure $<90 \mathrm{mmHg}$, no. (\%) & 0 \\
\hline Presenting oxygen saturation $<90 \%$, no. $(\%)$ & $3(4)$ \\
\hline $\begin{array}{l}\text { Presenting white blood cell count }>11,000 \text { cells } / \mathrm{mcl} \text {, } \\
\text { no. }(\%)\end{array}$ & $43(64)$ \\
\hline Serum procalcitonin $>0.25 \mathrm{ng} / \mathrm{ml}$, no. $(\%)$ & $36(54)$ \\
\hline ICU admission, no. (\%) & $14(21)$ \\
\hline Invasive mechanical ventilation, no. (\%) & $2(3)$ \\
\hline Vasopressor use, no. (\%) & $3(4)$ \\
\hline Hospital length of stay, median [IQR], days & $3[2-5]$ \\
\hline
\end{tabular}


Table 2 Cognitive Scores after Hospitalization for Pneumonia

\begin{tabular}{|c|c|c|}
\hline Outcome $^{\text {a }}$ & 2 months & 12 months \\
\hline \multicolumn{3}{|l|}{ RBANS scores ${ }^{b}$} \\
\hline Global cognition & $86[77,95]$ & $87[79,94]$ \\
\hline Impaired, no. (\%) & $14(25)$ & $13(23)$ \\
\hline Immediate memory & $94[83,106]$ & $97[85,109]$ \\
\hline Impaired, no. (\%) & $6(10)$ & $6(11)$ \\
\hline Delayed memory & $92[83,95]$ & $94[88,101]$ \\
\hline Impaired, no. (\%) & $8(14)$ & $5(9)$ \\
\hline Visuospatial function & $69[64,81]$ & $75[66,78]$ \\
\hline Impaired, no. (\%) & $37(64)$ & $33(58)$ \\
\hline Attention & $97[82,109]$ & $94[85,103]$ \\
\hline Impaired, no. (\%) & $6(11)$ & $6(11)$ \\
\hline Language & $96[88,104]$ & $92[84,99]$ \\
\hline Impaired, no. (\%) & $5(9)$ & $4(7)$ \\
\hline \multicolumn{3}{|l|}{ Other cognitive test scores } \\
\hline Trails A (attention) ${ }^{\mathrm{c}}$ & $45[37,50]$ & $47[38,53]$ \\
\hline Impaired, no. (\%) & $9(16)$ & $12(21)$ \\
\hline Trails B (task switching) ${ }^{\mathrm{c}}$ & $48[40,54]$ & $49[43,55]$ \\
\hline Impaired, no. $(\%)$ & $7(13)$ & $5(9)$ \\
\hline Tower achievement (planning) ${ }^{\mathrm{d}}$ & $10[9,12]$ & $11[9,12]$ \\
\hline Impaired, no. (\%) & $2(4)$ & $3(5)$ \\
\hline
\end{tabular}

Abbreviations: RBANS repeatable battery for the assessment of neuropsychologic status

${ }^{a}$ Presented as median [interquartile range] unless otherwise noted

${ }^{b} R B A N S$ scores are age-adjusted and have a population mean $\pm S D$ of $100 \pm 15$, with lower scores indicating worse cognition. Per published norms, $6.7 \%$ of the general population is expected to score below the cutoff we used to identify "impaired"

${ }^{c}$ Trails $A$ and $B$ scores are age-adjusted and have a population mean \pm $S D$ of $50 \pm 10$, with lower scores indicating worse cognition. Per published norms, $6.7 \%$ of the general population is expected to score below the cutoff we used to identify "impaired"

${ }^{d}$ Tower Achievement scores are age-adjusted and have a population mean $\pm S D$ of $10 \pm 3$, with lower scores indicating worse cognition. Per published norms, $6.7 \%$ of the general population is expected to score below the cutoff we used to identify "impaired"

follow-up, 20 (36\%) of 56 patients had deficits in two or more cognitive domains, and $9(16 \%)$ had deficits in three or more domains. Similarly, $16(29 \%)$ of 56 patients assessed at 12 months had deficits in two or more cognitive domains, and $9(16 \%)$ had deficits in three or more domains. Visuospatial function, attention, and memory were the most frequently

Table 3 Long-Term Cognitive Impairment after Hospitalization for Pneumonia

\begin{tabular}{|c|c|c|}
\hline Outcome & $\begin{array}{l}2 \\
\text { months }\end{array}$ & $\begin{array}{l}12 \\
\text { months }\end{array}$ \\
\hline No impairment, no. (\%) & $21(38 \%)$ & $26(46 \%)$ \\
\hline Mild cognitive impairment, ${ }^{\mathrm{a}}$ no. (\%) & $21(38 \%)$ & $17(30 \%)$ \\
\hline Amnestic, single domain & $0(0 \%)$ & $0(0 \%)$ \\
\hline Amnestic, multiple domains & $2(4 \%)$ & $0(0 \%)$ \\
\hline Non-amnestic, single domain & $15(27 \%)$ & $13(23 \%)$ \\
\hline Non-amnestic, multiple domains & $4(7 \%)$ & $4(7 \%)$ \\
\hline $\begin{array}{l}\text { Moderate/severe cognitive impairment, }{ }^{\mathrm{b}} \text { no. } \\
(\%)\end{array}$ & $14(25 \%)$ & $13(23 \%)$ \\
\hline Without IADL disability ${ }^{\mathrm{c}}$ & $12(21 \%)$ & $11(20 \%)$ \\
\hline With IADL disability ${ }^{\mathrm{c}}$ & $2(4 \%)$ & $2(4 \%)$ \\
\hline
\end{tabular}

Abbreviations: IADL instrumental activities of daily living, MCI mild cognitive impairment

${ }^{a}$ We classified patients as having mild cognitive impairment using standard definitions ${ }^{25}$ (see eTable 2)

${ }^{b}$ We classified patients as having moderate/severe impairment if their Repeatable Battery for the Assessment of Neuropsychological Status global cognition score was $<78$ (an established cutoff)

${ }^{c}$ We identified IADL disability using the Functional Activities Questionnaire impaired domains, whereas language and executive function/ planning were infrequently impaired (Table 2 ).

Though older patients ( $\geq 65$ years) with multiple comorbidities had the lowest RBANS global cognition scores throughout follow-up, younger patients without comorbid illness also had scores lower (i.e., worse) than expected based on population norms (Fig. 2). At both 2 and 12 months, moderate-tosevere cognitive impairment was most common among adults $\geq 65$ years old [4/13 (31\%) and 5/13 (38\%), respectively] but also affected those $<65$ years old $[10 / 43(23 \%)$ and 8/43 (19\%), respectively].

Mild cognitive impairment was also common, noted in more than one-third of subjects during each follow-up visit (Table 3). Of the 21 patients with mild cognitive impairment according to 2-month RBANS results, only two had evidence of mild cognitive impairment at baseline based on Short IQCODE responses completed in the hospital after the onset of CAP. Similarly, only 1 of the 21 with mild cognitive impairment at 12-month follow-up had impairment at baseline. Memory impairment was common, but mild cognitive impairment rarely met amnestic subtype criteria because patients with memory deficits typically had moderate-to-severe rather than mild cognitive impairment. Non-amnestic mild

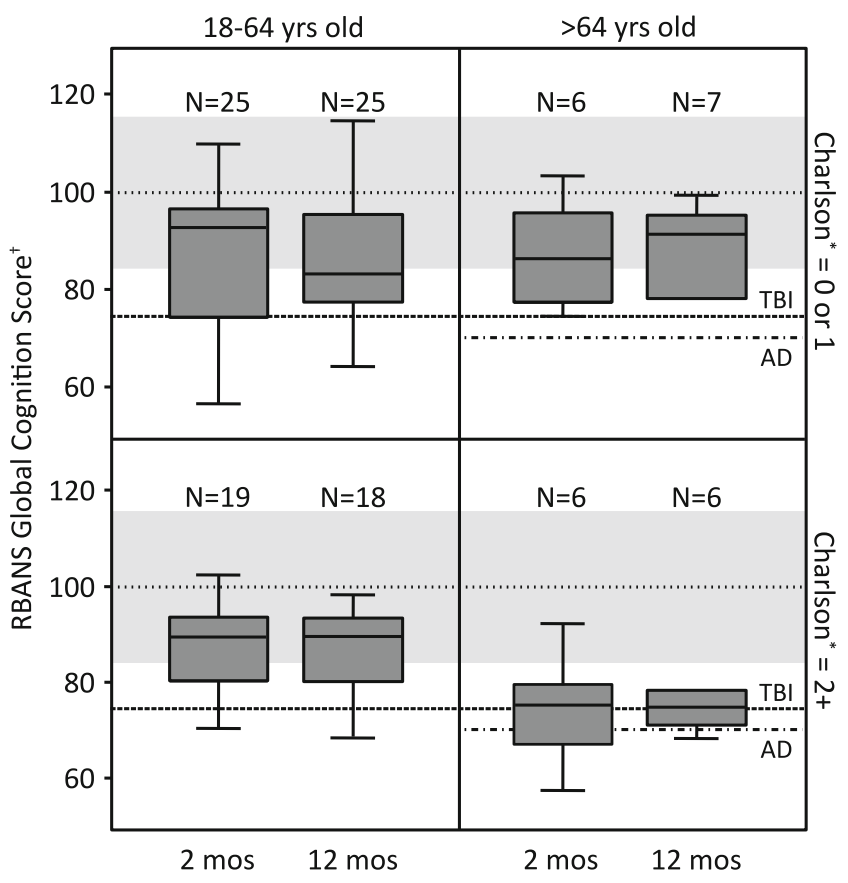

Figure 2 Cognitive function 2 and 12 months after communityacquired pneumonia according to age and comorbid illness burden per the Charlson Comorbidity Index. *The Charlson Comorbidity Index was measured at baseline; range $0-33,0=$ no comorbidities, 1 = one comorbidity. $\uparrow$ Repeatable Battery for the Assessment of Neuropsychological Status (RBANS) Global Cognition Score; ageadjusted population mean $\pm S D$ is $100 \pm 15$, with lower scores indicating worse cognition. The dashed line marked TBI indicates the expected population mean for moderate traumatic brain injury, and the dashed line marked AD indicates the expected population mean for mild Alzheimer's disease based on data collected in other cohort studies. The latter is shown only for patients $\geq 65$ years of age since RBANS population norms for AD were generated in that age group. 
cognitive impairment in a single domain was primarily due to visuospatial function deficits.

\section{DISCUSSION}

In this prospective cohort study, we found that one out of every four adults hospitalized with CAP had moderate-to-severe cognitive impairment that persisted at least a year after CAP and another third had mild cognitive impairment. The deficits in cognition were wide ranging and affected multiple domains. In addition, cognitive impairment was present in both older and younger adults, many of whom had no comorbid conditions prior to CAP, and was not limited to patients who were critically ill. These results highlight the need for future research examining mechanisms that may explain the relationship between pneumonia and long-term cognitive impairment as well as interventions that may prevent or mitigate this adverse long-term outcome.

The long-term cognitive impairment observed in our cohort likely has important effects. Though mild cognitive impairment, by definition, does not limit activities of daily living (e.g., eating, bathing, etc.), symptoms can include noticeable decrements in cognitive abilities used each day in driving, managing finances, and completing multistep tasks, e.g., preparing a meal or planning an event, etc. More severe cognitive impairment can, of course, lead to pronounced functional limitations. In the setting of moderate-to-severe cognitive impairment-identified in $25 \%$ of our cohort-financial errors, for example, would go beyond bookkeeping mistakes to involve errors in judgment, and problems cooking would involve not just poor planning but repeatedly forgetting to turn off the stove. Since our study reveals that middle-aged adults are at risk for post-CAP cognitive impairment, future studies should assess the impact of CAP hospitalization on patients' ability to return to work and/or their regular daily activities.

In addition to being consistent with results from earlier reports, our study extends previous observations by being the first to use a comprehensive cognitive battery to examine the frequency and characteristics of cognitive impairment in adults of all ages who survived a CAP hospitalization. Because we examined a population that was younger and less severely ill than those previously studied, our results suggest that the problem of post-acute illness cognitive impairment is more far reaching than previously thought. A number of studies have shown that up to half of patients who survive critical illness requiring mechanical ventilation struggle with significant cognitive impairment months to years after their hospitalization. ${ }^{14,16}$ In addition, Elhenbach et al. ${ }^{26}$ demonstrated that poor cognitive outcomes are not limited to just ICU patients; in their prospective cohort study of 2929 older adults without dementia at baseline, an acute care hospitalization was associated with a significant decline in cognitive function and was identified as an independent risk factor for incident dementia. CAP, one of the most common causes of such hospitalizations, may be particularly prone to cause declines in cognition via hypoxia, ${ }^{27,28}$ inflammation, ${ }^{29,30}$ and other mechanisms ${ }^{31}$ of organ dysfunction attributable to pneumonia.

Prior to our investigation, three studies examined whether older patients hospitalized for CAP had changes in cognition, but none of these studies were designed to evaluate characteristics of post-CAP cognitive impairment (e.g., specific cognitive domains, subtypes of mild cognitive impairment, etc.) nor did they examine younger adults with CAP. Davydow and colleagues $^{8}$ analyzed cognitive outcomes measured in the Health and Retirement Study to determine whether patients hospitalized with CAP during this long-term longitudinal study of patients $>50$ years of age had declines in cognition associated with CAP. After adjusting for multiple potential confounders, CAP hospitalization was associated with a 2.46-fold increase in the odds of moderate-to-severe cognitive impairment, which affected $25 \%$ of patients after CAP. Similarly, Shah and coworkers ${ }^{9}$ analyzed data from the Cardiovascular Health Study, a long-term longitudinal study of patients $\geq 65$ years of age and found CAP was associated with a 1.57fold increase in the hazard of dementia (diagnosed in $12 \%$ of CAP survivors) after adjusting for potential confounders. Finally, Tate et al. ${ }^{10}$ assessed healthy volunteers 75 years and older enrolled in a dementia trial and found that CAP hospitalization was associated with a 1.9-fold increase in the hazard of dementia. Importantly, each of these studies tested patients' cognition before and after hospitalization for CAP so that cognitive decline related to CAP could be identified.

In our study, we found that long-term cognitive impairment affecting survivors of CAP had a pattern of deficits similar to that seen in post-critical illness cognitive impairment (also known as critical illness brain injury) ${ }^{32}$; i.e., deficits were present in multiple domains of cognition. This pattern is more similar to that observed in traumatic brain injury than in indolent forms of cognitive impairment, e.g., Alzheimer's disease, which primarily affects memory until late in the disease. Whereas approximately one in four patients in our study had moderate-to-severe cognitive impairment 1 year after CAP, more than one in three survivors of critical illness had cognitive impairment at 1 year in a recent, large cohort study. ${ }^{16}$ Our study was not sufficiently powered to test whether the high severity of illness common to ICU patients is an independent risk factor for post-CAP cognitive impairment. Nevertheless, one potential explanation for our results is that threats to organ function common in severe critical illness-e.g., hypoxemia, inflammation-driven microvascular thrombosis, mitochondrial dysfunction, among others - may also be pathogenic mechanisms underlying cognitive impairment in survivors of pneumonia. Indeed, both hypoxia and inflammation are risk factors for cognitive impairment after sepsis and in other settings. ${ }^{27-30}$

Strengths of our study include the use of a rigorous definition of CAP to identify the study population, ${ }^{17}$ inclusion of both younger and older adults without evidence of preexisting 
cognitive impairment, and use of a well-validated instrument to evaluate patients for preexisting cognitive impairment. Additionally, though even more rigorous and in-depth batteries are available, we employed a widely used, comprehensive battery of cognitive tests, which allowed for detailed characterization of cognitive impairment as well as comparison with other populations, e.g., those with traumatic brain injury or Alzheimer's disease. Like most other investigations of acute illnesses, ${ }^{11-14,16}$ we were limited in our ability to directly assess baseline cognition since occurrence of CAP is rarely predictable. As done in prior studies, we therefore excluded patients with preexisting cognitive impairment severe enough to prevent independent living and relied on a validated questionnaire $^{18}$ to identify baseline mild cognitive impairment at time of CAP hospitalization. Despite these efforts, we cannot rule out the possibility that some proportion of the mild cognitive impairment observed in one-third of patients during follow-up was present prior to CAP. Indeed, our results suggest that $12 \%$ of participants had mild cognitive impairment prior to being hospitalized with CAP. Though delirium has been shown to predict long-term cognitive impairment after acute and critical illness, we were unable to study this relationship during the current investigation because delirium often goes undiagnosed when patients are not assessed systemically using a validated tool, which we did not employ during this study. Additionally, our study was not powered to analyze the relationship between long-term cognitive outcomes and delirium or other potential risk factors, which should be examined in future, larger investigations. Since patients $\geq 65$ years old and those mechanically ventilated were less likely to be enrolled in the EPIC study, ${ }^{17}$ our findings may underestimate the overall prevalence of cognitive impairment in CAP survivors. Since we enrolled patients at only one urban tertiary care center, it is unclear if these results are generalizable to other settings. Finally, a number of subjects withdrew or were lost to follow-up; though these subjects did not differ from those assessed in any measurable way, our results may have been biased if cognitive impairment was more (or less) likely among those not assessed during follow-up than among those evaluated.

In conclusion, long-term cognitive impairment, which varies in severity from mild to severe, is common in patients who were hospitalized for CAP, with moderate-to-severe impairment affecting one in four survivors 1 year after hospital discharge. Similar to ICU patients with respiratory failure requiring mechanical ventilation, $\mathrm{CAP}$ patients are at high risk for impairment in multiple domains of cognition, including memory, attention, and visuospatial function. In addition to examining mechanisms that may explain the relationship between long-term cognitive impairment and CAP, future studies should seek to identify potentially modifiable risk factors for this potentially life-altering outcome.

Corresponding Author: Timothy D. Girard, MD, MSCI; Clinical Research, Investigation, and Systems Modeling of Acute illness
(CRISMA) Center in the Department of Critical Care MedicineUniversity of Pittsburgh School of Medicine, Pittsburgh, PA 15261, USA (e-mail: timothy.girard@upmc.edu).

Funding This study was supported by a cooperative agreement with the Centers for Disease Control and Prevention (U18 IP000299) and by CTSA award no. UL1TR000445 from the National Center for Advancing Translational Sciences at the National Institutes of Health (NIH). Dr. Girard received support from the National Institutes of Health (NIH) (AG034257) and the Veterans Affairs (VA) Tennessee Valley Geriatric Research, Education, and Clinical Center (GRECC). Dr. Self was supported in part by a grant from the National Institute of General Medical Sciences (K23GM110469). Apart from the CDC, whose representatives worked with local investigators to design, conduct, analyze, and interpret the EPIC study, the other sponsors had no role in study design; data collection, analysis, and interpretation; or publication of results. The findings and conclusions in this report are those of the authors and do not necessarily represent the official position of the Centers for Disease Control and Prevention.

\section{Compliance with Ethical Standards:}

Prior Presentations: Some of the results of this study were previously reported in the form of an abstract at the American Thoracic Society 2015 International Conference on May 20, 2015.

Conflicts of Interest: The authors declare that they do not have a conflict of interest.

\section{REFERENCES}

1. Heron M. Deaths: leading causes for 2004. Natl Vital Stat Rep. 2007;56:1-95.

2. DeFrances CJ, Hall MJ. 2005 National Hospital Discharge Survey. Adv Data. 2007:1-19.

3. Pfuntner A, Wier LM, Stocks C. Most Frequent Conditions in U.S. Hospitals, 2011: Statistical Brief \# 162. Healthcare Cost and Utilization Project (HCUP) Statistical Briefs. Rockville 2006.

4. Fine MJ, Auble TE, Yealy DM, et al. A prediction rule to identify low-risk patients with community-acquired pneumonia. N Engl J Med. 1997;336:243-250

5. Mortensen EM, Kapoor WN, Chang CC, Fine MJ. Assessment of mortality after long-term follow-up of patients with community-acquired pneumonia. Clin Infect Dis. 2003;37:1617-1624.

6. Eurich DT, Marrie TJ, Minhas-Sandhu JK, Majumdar SR. Ten-Year Mortality after Community-acquired Pneumonia. A Prospective Cohort. Am J Respir Crit Care Med. 2015; 192:597-604.

7. Restrepo MI, Faverio P, Anzueto A. Long-term prognosis in community-acquired pneumonia. Curr Opin Infect Dis. 2013;26:151158.

8. Davydow DS, Hough CL, Levine DA, Langa KM, Iwashyna TJ. Functional disability, cognitive impairment, and depression after hospitalization for pneumonia. Am J Med. 2013;126:615-624 e615.

9. Shah FA, Pike F, Alvarez K, et al. Bidirectional relationship between cognitive function and pneumonia. Am J Respir Crit Care Med. 2013; 188:586-592.

10. Tate JA, Snitz BE, Alvarez KA, et al. Infection hospitalization increases risk of dementia in the elderly. Crit Care Med. 2014;42:1037-1046.

11. Hopkins RO, Weaver LK, Pope D, Orme JF, Bigler ED, Larson LV. Neuropsychological sequelae and impaired health status in survivors of severe acute respiratory distress syndrome. Am J Respir Crit Care Med. 1999; 160:50-56.

12. Jackson JC, Hart RP, Gordon SM, et al. Six-month neuropsychological outcome of medical intensive care unit patients. Crit Care Med. 2003;31:1226-1234.

13. Jackson JC, Girard TD, Gordon SM, et al. Long-term cognitive and psychological outcomes in the awakening and breathing controlled trial. Am J Respir Crit Care Med. 2010;182:183-191. 
14. Girard TD, Jackson JC, Pandharipande PP, et al. Delirium as a predictor of long-term cognitive impairment in survivors of critical illness. Crit Care Med. 2010;38:1513-1520.

15. Iwashyna TJ, Ely EW, Smith DM, Langa KM. Long-term cognitive impairment and functional disability among survivors of severe sepsis. JAMA. 2010;304:1787-1794.

16. Pandharipande PP, Girard TD, Jackson JC, et al. Long-term cognitive impairment after critical illness. N Engl J Med. 2013;369:1306-1316.

17. Jain S, Self WH, Wunderink RG, et al. Community-acquired pneumonia requiring hospitalization among U.S. adults. N Engl J Med. 2015;373:415-427.

18. Jorm AF, Jacomb PA. The Informant Questionnaire on Cognitive Decline in the Elderly (IQCODE): socio-demographic correlates, reliability, validity and some norms. Psychol Med. 1989; 19:1015-1022.

19. Katz S, Ford AB, Moskowitz RW, Jackson BA, Jaffe MW. Studies of Illness in the Aged. The Index of Adl: A Standardized Measure of Biological and Psychosocial Function. JAMA. 1963;185:914-919.

20. Pfeffer RI, Kurosaki TT, Harrah CH, Jr, Chance JM, Filos S. Measurement of functional activities in older adults in the community. J Gerontol. 1982;37:323-329.

21. Charlson ME, Pompei P, Ales KL, MacKenzie CR. A new method of classifying prognostic comorbidity in longitudinal studies: development and validation. J Chronic Dis. 1987;40:373-383.

22. Randolph C. Repeatable Battery for the Assessment of Neuropsychological Status (RBANS) Manual. San Antonio: Psychological Corporation; 1998.

23. Lezak MD. Neuropsychological Assessment. 3rd ed. New York: Oxford University Press; 1995.
24. Delis DC, Kaplan E, Kramer JH. Delis-Kaplan Executive Function System (D-KEFS): Examiner's Manual. San Antonio: Psychological Corporation; 2001

25. Petersen RC. Clinical practice. Mild cognitive impairment. N Engl J Med. 2011;364:2227-2234.

26. Ehlenbach WJ, Hough CL, Crane PK, et al. Association between acute care and critical illness hospitalization and cognitive function in older adults. JAMA. 2010;303:763-770.

27. Taccone FS, Su F, De Deyne C, et al. Sepsis is associated with altered cerebral microcirculation and tissue hypoxia in experimental peritonitis. Crit Care Med. 2014;42:e114-122.

28. Yaffe K, Laffan AM, Harrison SL, et al. Sleep-disordered breathing, hypoxia, and risk of mild cognitive impairment and dementia in older women. JAMA. 2011;306:613-619.

29. Murray C, Sanderson DJ, Barkus C, et al. Systemic inflammation induces acute working memory deficits in the primed brain: relevance for delirium. Neurobiol Aging. 2012;33:603-616 e603.

30. Yaffe $\mathbf{K}$, Kanaya A, Lindquist $\mathbf{K}$, et al. The metabolic syndrome, inflammation, and risk of cognitive decline. JAMA. 2004;292:2237-2242.

31. d'Avila JC, Santiago AP, Amancio RT, Galina A, Oliveira MF, Bozza FA. Sepsis induces brain mitochondrial dysfunction. Crit Care Med. 2008;36: 1925-1932.

32. Girard TD, Dittus RS, Ely EW. Critical illness brain injury. Annu Rev Med. 2016:67:497-513. 